\title{
Projeto Ágil de Aplicativo como Mediação da Aprendizagem sobre Orientação a Objetos
}

\author{
Walber A. R. Beltrame1 \\ ${ }^{1}$ Instituto Federal do Espírito Santo (IFES) - Campus Guarapari \\ Alameda Francisco Vieria Simões, 720 - Aeroporto, Guarapari - ES, 29216-795 \\ walber. beltrame@ifes.edu.br

\begin{abstract}
The studies about paradigm of Object Oriented Programming in curricular components of exact science courses are planned in sequence to learning of structured logic, as advance of knowledge for this model of computational solution. This transition is complex and requires that a set of skills and competences on programming structures be rebuilt, as to of evidencing differences, advantages and facilities such as overlap, inheritance, abstraction, overload, polymorphism, encapsulation, and design patterns. The aim of this article is to present a dynamic based on learning by real projects using agile principles as mediation of learning for this discipline, according to constructivist orientation. Discussions about model, tools and challenges are presented from an preliminary case study.
\end{abstract}

Resumo. Os conteúdos de paradigma de Programação Orientado a Objetos nos componentes curriculares dos cursos de ciências exatas são planejados em sequência ao aprendizado de lógica estruturada, como avanço dos saberes para esse modelo de solução computacional. Essa transição é complexa e requer que um conjunto de habilidades e de competências sobre estruturas de programação sejam reconstruídas, ao ponto de evidenciar diferenças, vantagens e facilidades, como herança, abstração, sobreposição, sobrecarga, polimorfismo, encapsulamento e padrões de projeto. O objetivo deste artigo é apresentar uma dinâmica baseada em aprendizagem por projetos reais usando princípios ágeis como mediação da aprendizagem dessa disciplina, por um viés construtivista. Discussões sobre o modelo, ferramentas e desafios são apresentados a partir de um estudo de caso preliminar.

\section{Introdução}

A aprendizagem por projetos caracteriza-se por metodologias baseadas em investigação, em propostas de tarefas e de desafios para solucionar situações-problema não triviais, integrando diferentes áreas do conhecimentos e estimulando o exercício de habilidades sobre competências, trabalho em grupo, protagonismo e pensamento crítico. A gestão do projeto coloca-se como um fator importante para que as atividades sejam planejadas e que a condução das práticas seja harmoniosa, de modo que se cumpram os prazos e as entregas de solução.

Em Bittencourt et al. (2013), uma experiência em computação é relatada como positiva para competências como comunicação, colaboração, liderança, autodidatismo, raciocínio crítico e aplicação dos conteúdos a situações práticas. No entanto, problemas típicos de software e o excesso de conceitos entre três disciplinas integradas geraram dificuldades no processo, afetando a motivação e aumentando as desistências. 
VII Congresso Brasileiro de Informática na Educação (CBIE 2018)

Anais dos Workshops do VII Congresso Brasileiro de Informática na Educação (WCBIE 2018)

Em Fagundes et al. (2006), o modelo de projeto para aprender deve ser baseado em conflitos, perturbações no sistema de significações, que constituem o conhecimento particular do aprendiz, denominado de Projetos de Aprendizagem. Uma diferença sútil das abordagens é que, enquanto no anterior Projeto conduz situações de Aprendizagem, no último Aprendizagem é que conduz o que será feito no Projeto. Essa alteração de lidar com os conceitos (Projeto e Aprendizagem) influencia em dois critérios centrais: (1) foco no aprendiz, sujeito repleto de conhecimentos e de incertezas; (2) a motivação de aprender é instigada na relação entre os desafios expostos, a satisfação pessoal, a vivência particular e a noção de superar o que já é conhecido. Porém, propostas abertas de projetos para aprendizagem podem ser complexas de serem geridas. Como fazer para aproximar essas abordagens?

O objetivo deste artigo é apresentar uma dinâmica baseada em aprendizagem por projetos reais usando princípios ágeis como mediação da aprendizagem da disciplina de Programação Orientada a Objetos, por um viés construtivista, de maneira a atenuar essa questão. A justificativa é que alinhar práticas de aprendizagem planejadas em função do aluno com as ações de aprender pelas práticas do projeto pode ser mais motivador e equilibrado, diante das expectativas do Projeto e do Aprendiz. Para tanto, na próxima seção, tem-se os referenciais teóricos sobre as exposições das seções seguintes.

\section{Referencial teórico}

Projetos e atividades planejadas em espaços colaborativos estão sendo propostos para educação, conforme observado em Carvalho et al. (2005) e Borges et al. (2017), como forma de proporcionar aos alunos ações personalizadas e avançadas de criação e de criatividade, apoiado por tecnologia, informática e pensamento computacional, para educação a distância ou em laboratórios presenciais.

Para este trabalho, considera-se Aprendizagem como uma ação de desequilíbrio epistêmico e ressignificação [Piaget, 1929-1979], base para o modelo (Seção 3). Assim, Mediação da Aprendizagem é o conjunto de interações que manifestam essa ação e as inúmeras possibilidades dessas interações [Vygotsky, 1978]. Os orientadores podem ter atitudes negativas ou positivas para esse complexo sistema [Human-Vogel \& Bouwer, 2005], por exemplo, apresentar soluções das dúvidas surgidas no desequilíbrio (ação negativa) ou solicitar que o aluno idealize, faça hipóteses para a solucionar as dúvidas e utilize de pesquisa científica para validá-las (ação positiva).

\section{Metodologia}

Para este estudo, optou-se pela criação e descrição de um modelo teórico que fornecesse orientação didática sobre a disciplina de Programação Orientada a Objetos e atividades interdisciplinares entre períodos letivos, adaptado de Nunes et al. (2017) e Medeiros et al. (2017), caracterizando-se como abordagem interpretativista (Seção 4). Para esta proposta descritiva, o modelo é exposto como conjunto de atividades organizadas entre gestão ágil de projeto, construção de aplicativo para dispositivos móveis e ações de mediação da aprendizagem para as competências desejadas.

A fim de observar o modelo na prática, um estudo de caso foi escolhido como instrumento de investigação (Seção 5), de profundidade exploratória preliminar e de caráter qualitativo, aplicado na disciplina de Linguagem de Programação de um curso superior de Engenharia Elétrica, em que o conteúdo de Orientação a Objetos é um dos componentes curriculares. 
VII Congresso Brasileiro de Informática na Educação (CBIE 2018)

Anais dos Workshops do VII Congresso Brasileiro de Informática na Educação (WCBIE 2018)

A disciplina semestral foi conduzida na forma de projetos, em que o propósito era atender requisitos reais a partir de um programa de pesquisa e de extensão entre a instituição superior e uma autarquia estadual de desenvolvimento de software. São brevemente apresentadas as discussões sobre o modelo, ferramentas e desafios iniciais encontrados.

\section{Modelo de Projeto}

Para fins de compreensão do modelo de Projeto proposto neste artigo (Figura 1), divide-se o processo didático em três grandes componentes: (1) Gestão de Projetos, em que são incentivados técnicas segundo os princípios ágeis e ferramentas de trabalho, como o método Scrum [Medeiros et al. 2017]; (2) Fases Práticas, em que se equilibram os planejamentos e procedimentos ágeis com as atividades de construir e aprender, a partir de soluções reais para um problema desafiador [Nunes et al. 2017]; (3) Mediação da Aprendizagem, em que um conjunto de atividades cíclicas são propostas para promover desequilíbrios epistêmicos e reconstrução dos saberes sobre as habilidades desejadas, no caso, sobre Programação Orientada a Objetos.

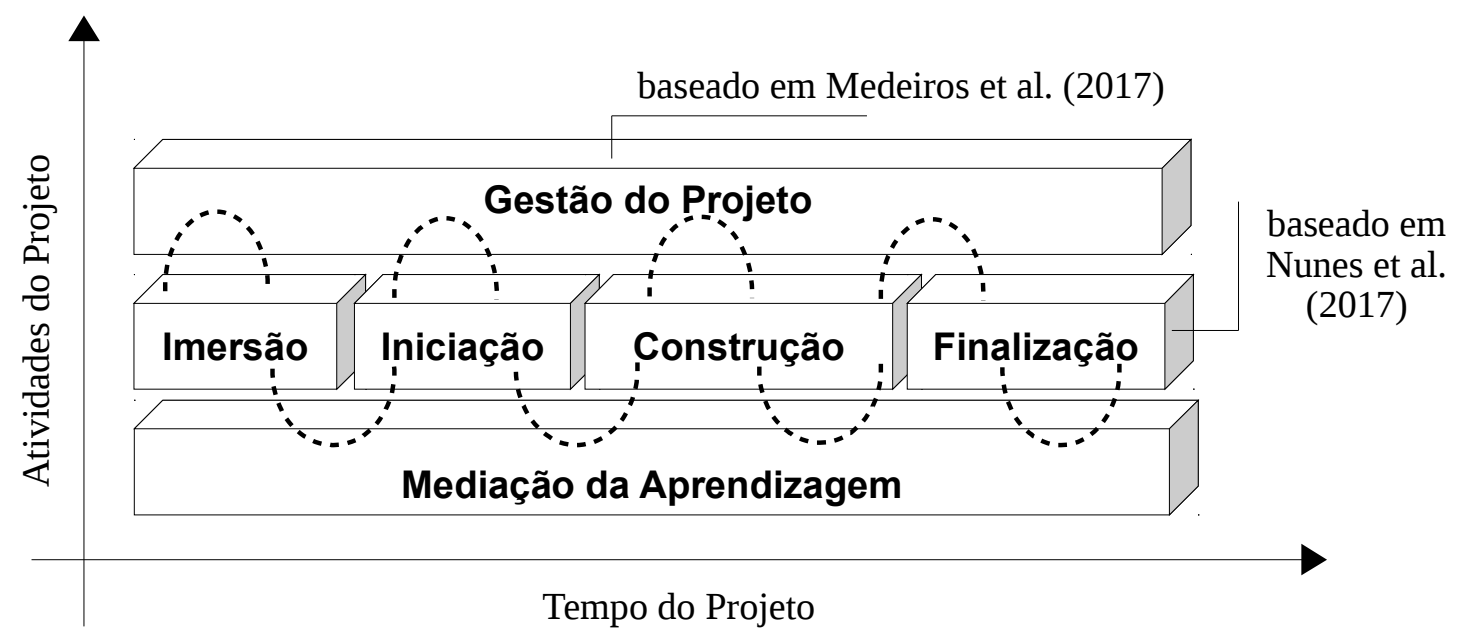

Figura 1. A Onda Ágil no gráfico Tempo versus Atividades do Projeto

As Atividades do Projeto, em cada componente, decorrem no Tempo, de modo que formam uma Onda no gráfico, alternando questões de gestão, de prática e de aprendizagem, mas, todas contribuem para se ter competências aprendidas (Figura 2).

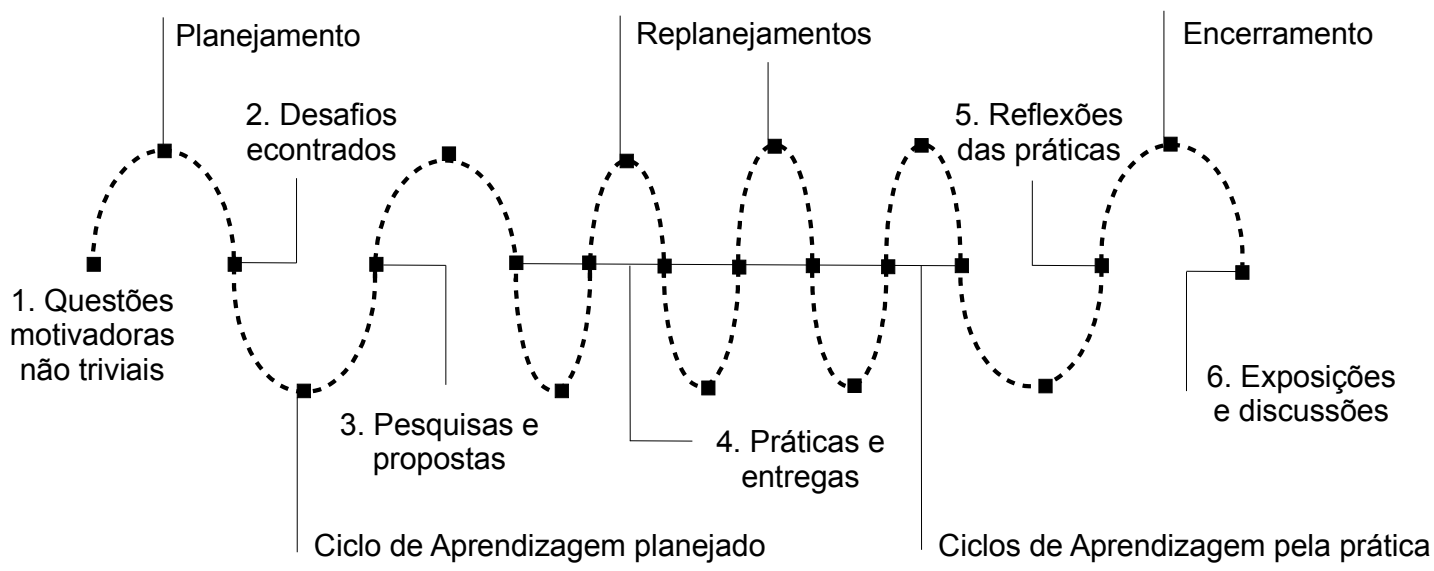

Figura 2. Detalhamento da Onda Ágil e os Ciclos de Aprendizagem 
Quanto à Gestão do Projeto, cada atividade prática é planejada e replanejada pelos orientadores, segundo diretrizes ágeis, como entregas parciais (sprints), reuniões constantes e feedbacks. Deve-se encontrar problemas não triviais e relacionados ao contexto possível a ser explorado pelos estudantes e que despertem interesse, autonomia e curiosidade [Freire, 1998]. Esses desafios são estímulos para ações direcionadas de aprendizagem quanto às Fases Práticas:

1. Questões Motivadoras não triviais: por meio de programas de pesquisa e de extensão universitária, comuns em cursos superiores, pode-se fazer listagens de possíveis problemas reais da sociedade e apresentar aos alunos na forma de ideias iniciais de aplicativos como solução. Os aplicativos móveis demandam interfaces mais simples de software (limitação dos aparelhos), que por sua vez levam a estrutura de dados também não complexas, ideal para uma transição entre os paradigmas Procedural e Orientado a Objetos. Após escolhas do tema pelos estudantes, deve-se fazer reuniões com as comunidades envolvidas. Os desafios devem ser apresentados por todos, assim como assuntos relacionados, de forma que os encontros motivarão empatia entre o grupo;

2. Desafios encontrados: nessa etapa, realizam-se pesquisas e análises de requisitos com essa comunidade, por meio de modelos rápidos e quadros que descrevem o problema, as pessoas e o projeto. Esses modelos descritivos auxiliarão quanto aos requisitos funcionais e não funcionais do aplicativo. Se possível, os alunos devem fazer uma imersão, ou seja, devem sentir os problemas, ver de perto e até trabalhar por um tempo junto às pessoas, fazerem filmagens ou anotações, ao ponto de se sensibilizarem com o que de fato acontece;

3. Pesquisas e propostas: nessa fase, os alunos são orientados a pesquisar soluções existentes, avaliarem essas soluções, ver pontos fortes e fracos. Devem propor ideias para o aplicativo, fazer e refazer rascunhos para as telas, pesquisar teorias sobre como modelar o aplicativo usando Orientação a Objetos e validar com os professores as propostas. A partir dessas definições, planejamentos, divisões de tarefas e priorizações sobre as práticas de construção e de programação do aplicativo são iniciadas (sprints);

4. Práticas e entregas: nesse momento, responsabilidades e tarefas para criação do aplicativo são executadas na forma de sprints do método Scrum [Medeiros et al. 2017] e, a cada quinzena, os alunos e os orientadores reúnem-se para discutir as entregas e evoluções, além de tirar dúvidas. Considera-se que essas tarefas são interações de aprendizagem pelas práticas individuais e coletivas. Anotam-se detalhes importantes em diários de bordo e avaliação é feita tanto pela qualidade das entregas quanto pelas atitudes comportamentais;

5. Reflexões das práticas: durante essa etapa, os alunos disponibilizam o aplicativo para testes e são orientados a elaborar questionários avaliativos. A partir da análise de respostas dos participantes, debatem sobre o trabalho desenvolvido, apresentam pontos positivos e negativos, principais dificuldades e resultados perante aos desafios iniciais propostos;

6. Exposições e discussões: nesse final, submete-se o aplicativo para exposições, desde mostras municipais e estaduais de tecnologia, até eventos científicos de nível nacional, a fim de estimular comunicação interpessoal, as lideranças e as habilidades de diálogo com a sociedade, com novas possibilidades de melhoria e de correções. 
VII Congresso Brasileiro de Informática na Educação (CBIE 2018)

Anais dos Workshops do VII Congresso Brasileiro de Informática na Educação (WCBIE 2018)

Segundo a perspectiva piagetiana norteadora deste trabalho, uma sequência de atividades são propostas como mediadoras da Interação nas fases do projeto (Figura 3), que se denominou Ciclo de Aprendizagem (iniciativa deste artigo), considerando que Competência é o objetivo do estudo sobre Programação Orientada a Objetos e que ela exerce uma força perturbadora sobre os alunos, de forma a envolvê-los em novas ações de compreensão. Entende-se que as habilidades esperadas a partir dessa competência são as cognições que os permitirão demonstrar domínio sobre os conteúdos, de forma significativa ao longo do tempo.

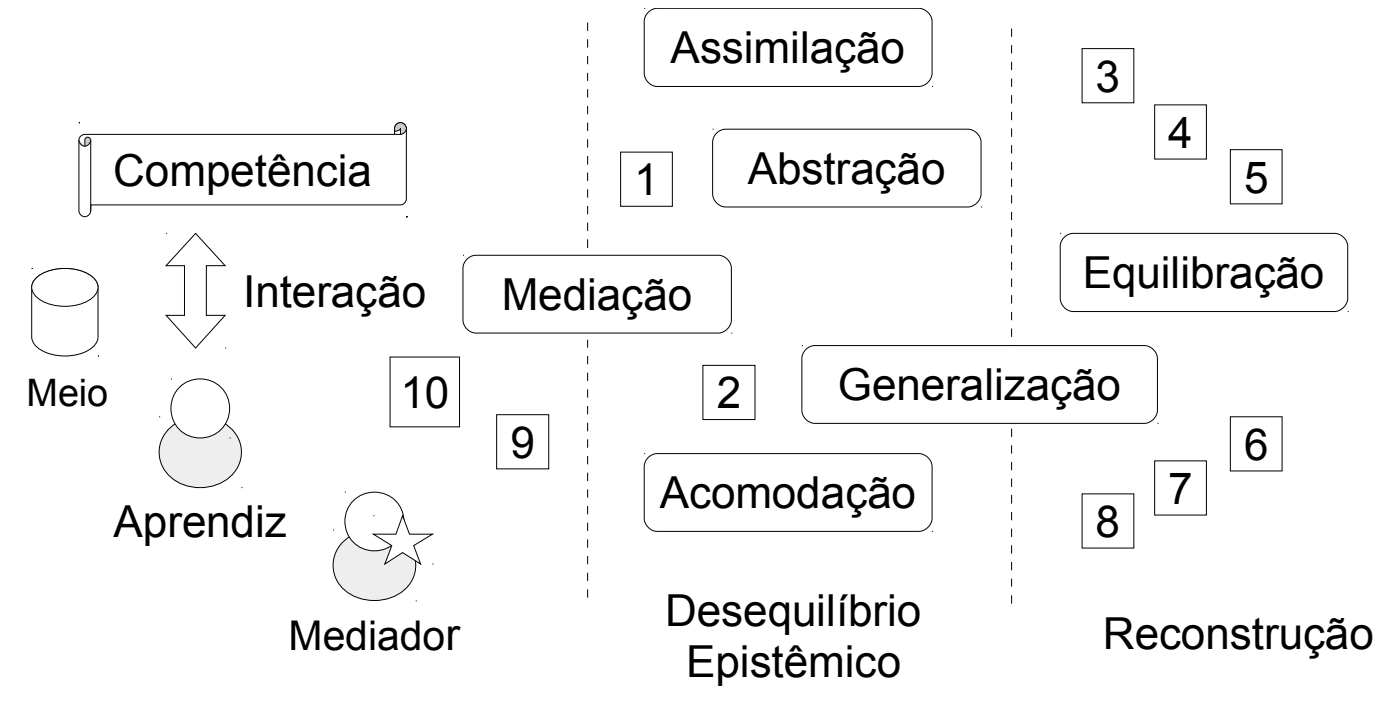

Figura 3. Atividades do Ciclo de Aprendizagem proposto para o Projeto

A partir da concepção de aprendizagem como um processo de desequilíbrio epistêmico e ressignificação, a mediação da aprendizagem é conjunto de interações entre o indivíduo e o meio que permitem que esse processo aconteça, considerando aspectos inerentes como o conhecimento anterior, a habilidade de interpretação do indivíduo e as inúmeras possibilidades de manifestação dessas interações. Coloca-se exposto, então, três elementos importantes: os indivíduos aprendizes, os mediadores (naturais ou sintéticos) e o meio (ambiente, objetos, comunicação, linguagens, espaços de interações). Apesar dessa separação, para fins de entendimento, esses elementos podem não estar claramente desassociados, pois um mediador pode ser um indivíduo aprendiz, o aprendiz fazer parte do meio, o modificar e o interpretar diferentemente dos outros indivíduos, o mediador influenciar nessas interpretações e o meio também pode ser o indivíduo aprendiz e o mediador.

Durante o desequilíbrio, considera-se Assimilação a tentativa do indivíduo em solucionar as situações impostas pela interação por meio da estrutura cognitiva atual, considerando todas as experiências vivenciadas até o momento, ao que se chama de esquemas. No entanto, essa estrutura pode não ser suficiente para compreender a nova realidade, disparando o processo de Acomodação, que é a tentativa de modificar esses esquemas mentais antigos para dominar a recente competência, por exemplo, com atitudes de buscar, desvendar, pesquisar, correlacionar, solucionar e esquematizar novamente esse desequilíbrio.

Durante o movimento cognitivo para assimilar informações importantes da competência a partir da interação, realiza-se o processo de Abstração, que é a operação mediante a qual alguns detalhes são escolhidos como objeto de percepção, a fim de 
isolar escolhas e assumir como especificidades, seja na prática empírica de observação das qualidades dos objetos e das ações, seja na capacidade de refletir sobre como essas qualidades correlacionam. Dessa forma, pode-se ler dois parágrafos de um texto e selecionar as palavras mais importantes para saber sobre o que elas tratam (qualidades observáveis) e correlacionar os sentidos entre esses termos (qualidades reflexionantes).

No esforço de acomodar os objetos abstraídos, passa-se por um outro processo cognitivo de Generalização, que é a operação de integrar as informações relevantes às totalidades existentes, a fim de categorizar, classificar, encontrar similaridades, não somente ao contexto específico da interação, mas a todos outros esquemas formados, induzindo as abstrações às formas descontextualizadas, do particular ao universal. $\mathrm{Na}$ Orientação a Objetos, faz-se comumente essas operações quando se modela os objetos em classes, classes em heranças, heranças em interfaces. Portanto, a própria atividade de Programação Orientada a Objetos é rica desse complexo movimento entre abstrair e generalizar para se aprender sobre a realidade.

Complementar a essas ações, tem-se que a Equilibração ou Reconstrução do conhecimento se manifesta em quatro fatores principais: (1) combinação dos elementos de interação, comunicação social, maturação e experiência; (2) os processos biológicos intrinsecamente característicos da espécie humana; (3) a capacidade do meio em que o aprendiz está inserido em ofertar novas interações ainda mais ricas; (4) a experiência social do aprendiz em relação aos mediadores e os ambientes em que este já fora oportunizado.

Doravante a esse segundo item e de acordo com os estágios de desenvolvimento piagetianos, um adolescente a partir dos doze anos é favoravelmente capaz de raciocinar formalmente, ou seja, consegue traçar hipóteses, deduzir consequências e implicações. No entanto, contextos desfavoráveis fazem com que esse pensamento seja constituído somente em fase adulta, até os vinte anos. A partir desse estágio, os estudantes podem desenvolver melhor as habilidades para lidar com programação. Na fase universitária, após terem passado por atividades de lógica estruturada, tendem a compreender sobre paradigma de Orientação a Objetos satisfatoriamente.

Entretanto, alguns aspectos práticos e situações típicas de softwares complexos, em que as competências de Orientação a Objetos tornam-se mais evidentes, podem não ser de fácil simulação pelos professores ou exigirem que exercícios com grandes cargas de trabalho sejam propostos aos alunos dessa disciplina. $\mathrm{O}$ aprendiz pode, interagindo com o meio e o mediador, realizar um esforço mental de imaginação dos problemas simulados e realizar interações, mas, esse alto esforço pode ser fator de desmotivação, agravado quando feito de forma isolada. Em contraste, por já possuírem habilidades em programação, os jovens desejam avançar nas práticas da carreira profissional. Assim, a aprendizagem por projetos reais e por metodologias ágeis incrementam um componente de estímulo a essa perspectiva.

Desse modo, como alinhar abordagens de aprendizagem por projetos e planejar interações voltadas ao desequilíbrio epistêmico e à reconstrução? Por exemplo, como fazer para que um aluno deixe de resolver um problema computacional usando somente a lógica estruturada e use corretamente todas as boas práticas da Orientação a Objetos? Sabendo que nem todos os projetos necessitam das competências necessárias para lidar com essas práticas. Um projeto de software em que o conceito de sobrecarga (quando um mesmo nome de método executa procedimentos e resultados de tipos diferentes) não seja necessário, como fazer com que essa competência seja estudada? 
Para tanto, este artigo recomenda que o mediador planeje interações aos alunos para todas competências desejadas, mesmo que se crie requisitos e funcionalidades ao projeto de software. Deve-se também evitar interações baseadas somente em exposições de conteúdo, mas provocar desequilíbrios e desafios, de forma planejada. A partir disso, este artigo propõe um conjunto de atividades (Ciclo de Aprendizagem planejado) a cada marco do projeto pelo mediador:

1. Apresentar um desafio, uma solução e deficiências decorrentes, que sejam entendidos pelo estudante e que sejam relacionados aquela fase do projeto. Por exemplo, dado um aluno iniciante em Orientação a Objetos e que o requisito do projeto é fazer um cálculo. Assim, apresente outro desafio similar de cálculo, uma solução do cálculo na forma de lógica estruturada e algumas deficiências, por exemplo, um grande número de variáveis. Nesse ponto, entende-se que o aprendiz realiza uma atividade de Assimilação;

2. Apresentar possíveis caminhos a essas deficiências e solicitar estudos (fazer leituras, assistir videos, procurar exemplos de soluções, interagir com objetos de aprendizagem mais elaborados), de modo que produzam um quadro de dúvidas temporárias e certezas provisórias (quadro cognitivo). Do exemplo, comentar que os códigos do paradigma de Orientação a Objetos organizam melhor as variáveis, por causa do encapsulamento. Os alunos deverão construir materiais sobre isso e fazer uma postagem pública a todos estudantes. Nesse momento, entende-se que o aprendiz inicializa a atividade de Acomodação;

3. Solicitar ao aluno resolver o desafio com os novos caminhos estudados. Ainda que o aluno resolva o desafio parcialmente ou com exigências de qualidade não cumpridas, é necessário que ele faça uma tentativa e demonstre ao mediador o que fora pensado, a partir daquilo que se compreendeu nos estudos. Percebe-se que o aprendiz tentará reequilibrar os conhecimentos dessa competência para resolver o desafio. Ainda no exemplo anterior, o aluno tentaria construir uma ou mais classes para calcular o resultado esperado;

4. Demonstrar ao aprendiz algumas boas práticas e erros comuns que aqueles caminhos orientam, com outros exemplos que não o primeiro desafio, mas que se assemelhe a ele. Deve-se questionar os estudantes se essas boas práticas e erros comuns também acontecem na solução apresentada até o momento;

5. Solicitar aos alunos interações em grupo sobre as soluções apresentadas do desafio inicial, com finalidade de se avaliarem, concordarem ou discordarem, verem respostas semelhantes e diferentes, se as boas práticas e os erros comuns se manifestam e de que forma se apresentam na solução do estudante comparado com os demais;

6. Avaliar se boas práticas (presentes e faltantes) e erros comuns foram listados de forma bem estruturada no debate, apontar inconsistências, mostrar aquelas que não foram bem discutidas nos grupos, usando de novos exemplos (se possível);

7. Solicitar aos alunos resolverem novamente o desafio inicial, agora usando como base as boas práticas e evitando os erros comuns. No exemplo, refazer as classes que faziam os cálculos de forma a evitar um erro comum de programação;

8. Avaliar a qualidade da solução encontrada a partir de parâmetros estabelecidos, individualmente e coletivamente. Considerar também todo o processo do aluno, como o grau de envolvimento em grupo e questões comportamentais; 
VII Congresso Brasileiro de Informática na Educação (CBIE 2018)

Anais dos Workshops do VII Congresso Brasileiro de Informática na Educação (WCBIE 2018)

9. Solicitar aos alunos um relato da experiência desse ciclo de aprendizagem, por exemplo, fazer uma postagem sobre o aprendizado, gravar um vídeo, construir um tutorial, fazer um mapa conceitual ou outras formas de representação do que foi aprendido, principalmente, o quadro cognitivo;

10. Pedir para que os estudantes avaliem os relatos dos colegas quanto à forma $\mathrm{e}$ quanto aos conteúdos, ou seja, se o outro estudante usou de boas maneiras na comunicação e os estudos relatados estão condizentes com o que fora realizado. As avaliações podem ser na forma de comentários ou outro meio estruturado.

O aluno deverá evoluir o quadro cognitivo (etapas 2 e 9), de maneira que ao final, ele será questionado durante o Cliclo de Aprendizagem pela prática quanto a habilidade para realizar aquela competência no projeto, agora como desafio real. Essa atividade mediada poderá ser refeita quantas vezes necessário, por isso cíclica, no caso do professor avaliar se ainda não houve compreensão das capacidades necessárias para domínio em questão. Esse fato impacta nos replanejamentos do projeto quanto a prazos.

\section{Estudo de caso}

A partir de reuniões anteriores a oferta da disciplina Linguagem de Programação para o curso superior de Engenharia Elétrica, advindas de um programa de extensão, entre o instituto de educação e a autarquia pública de desenvolvimento de software, voltado a construção de micro serviços para o aplicativo móvel do governo estadual, com suporte a Cidades Inteligentes (Smart Cities, em inglês), listou-se uma série de possibilidades para projetos: (1) notificações sobre compras e abertura de preços de órgãos públicos; (2) agendamento de serviços presenciais; (3) mapa sobre a acessibilidade dos locais públicos para portadores de necessidades especiais; (4) acompanhamento das obras públicas por localidade; (5) alertas emergenciais (incêndios e calamidades); (6) boletim, frequência e calendário escolar; (7) comunicação entre escolas e responsáveis de alunos; (8) apoio a segurança pública por tipos de ocorrências.

Todas as possibilidades são demandas reais dos órgãos do governo, em que os processos correspondentes não possuem funcionalidades no aplicativo. Dessa forma, todas as secretarias foram contatadas e se prontificaram a participar do trabalho escolar, caso fossem escolhidas pelos alunos. Iniciado o período, a turma possuía vinte e três alunos, aprovados no semestre anterior em Algoritmos, ementa de lógica estruturada e linguagem C. Fora exposto o modelo do Projeto para o semestre, com carga horária de oitenta horas mensais (vinte semanas, de quatro aulas semanais), com as competências de se utilizar estruturas de dados mais comuns na construção de soluções, como vetores, matrizes e listas, fundamentados em Orientação a Objetos.

Exposto a primeira atividade prática, os estudantes formaram cinco grupos, com quatro a cinco alunos, e justificaram a escolha dos projetos. Devido a dificuldades de locomoção, os contatos com as secretarias se deram por e-mails ou videoconferências, intermediadas pelo professor. Um grupo preferiu, dentre o escopo da temática, fazer um projeto em parceria com microempresários locais (mercearias), para um aplicativo de compra rápida de mantimentos, sem sair de casa. Os outros projetos foram (2), (5), (7), (8 - para casos de agressão a mulher). O projeto (1) foi escolhido como modelo de referência da disciplina, utilizado pelo docente como projeto-exemplo para as atividades de Mediação da Aprendizagem. Fora explicado que o resultado do projeto não precisava ser funcional, ou seja, fazer transações reais e ser entregue como produto final aos órgãos, dado que certos conceitos não eram do escopo, como banco de dados. 
Para criação do aplicativo foram escolhidos a linguagem C\# (pela similaridade com C) e o Xamarin Forms como plataforma de desenvolvimento. Na imersão, além de trocas de correspondências eletrônicas com as secretarias estaduais, nas quatro semanas iniciais os alunos fizeram atividades para familiarização de ferramentas (noção básica): Github (compartilhamento de código), Moodle (ambiente de aprendizagem), Slack (recurso de comunicação), Taiga (gestão de projeto), Balsamiq (prototipagem) e Astah (diagrama de classes). Na iniciação, os estudantes fizeram pesquisas de aplicativos e avaliações, criaram os modelos principais do aplicativo e validaram com os órgãos, além das atividades planejadas de aprendizagem, durante quatro semanas.

$\mathrm{Na}$ construção, toda semana era desenvolvida uma tela planejada, em que foram identificadas competências específicas de Estrutura de Dados e de Orientação a Objetos, como encapsulamento, herança, abstração, sobreposição, sobrecarga, polimorfismo e padrões de projeto, com duração de oito semanas. Em paralelo e como novas atividades extraclasse, exercícios e interações do Ciclo de Aprendizagem planejado eram feitos. Mas, cada grupo teve uma sequência diferente, dependendo da tela e das habilidades necessárias traçadas para cada tela, a fim de trabalharem boas práticas e erros comuns. Por fim, nas quatros semanas finais, os alunos tiveram que preparar uma apresentação do que haviam realizado numa Mostra de Software, com todos envolvidos, desde o instituto, equipe técnica da autarquia e gestores públicos.

\section{Resultados e discussão}

Apesar de um escopo menor e carga horária reduzida em comparação a outros relatos [Bittencourt et al. 2013], [Nunes et al. 2017], [Medeiros et al. 2017], considera-se que um futuro detalhamento deste estudo de caso poderá demonstrar melhor a aplicação do modelo. No entanto, algumas lições aprendidas podem ser listadas: (1) notou-se boa motivação dos estudantes com a proposta de projetos ágeis de aplicativos baseados em casos reais e com a interação das entidades externas, visto que somente dois alunos desistiram e todos os outros foram aprovados com avaliação positiva das habilidades; (2) usar de exemplo um dos projetos ajudou o professor a propor atividades similares às escolhidas pelos alunos, porém, muitas telas ficaram semelhantes ao do docente, assim, pode-se concluir que se limitou a criatividade para as soluções idealizadas; (3) ao usar de várias ferramentas, houve um grande esforço de configuração, que por vezes era necessária intervenção do professor, gerando descontentamento dos alunos.

Ainda que os objetivos do experimento fossem os conteúdos introdutórios de Orientação a Objetos como evolução do paradigma procedural, observou-se que outras capacidades foram estudadas, como a utilização de linguagem de marcação XAML para a camada visual, com padrão de projeto MVVM (Model-View-ViewModel), que atua como intermediário entre os dados (modelo) e a interface do usuário (visualização) por meio de associação de dados e comandos. Para a persistência dos dados, dois grupos estudaram sobre ORM (Object Relational Mapping) com SGBD em servidor externo.

Como estudo preliminar, pode-se apontar também alguns méritos para futuros trabalhos quanto avaliação quantitativa e aprofundada do modelo, além de critérios de análise em novos experimentos: (1) índices de eficiência das soluções apresentadas em relação aos requisitos de software e de competências desejadas; (2) demonstração mais detalhada de artefatos produzidos nos futuros casos de experiência, a fim de evidenciar se as teorias piagetianas foram abservadas; (3) avaliação de ferramentas automáticas, como as de qualidade de código, de acompanhamento nos ambientes de programação e tutores inteligentes para melhoria das interações. 
VII Congresso Brasileiro de Informática na Educação (CBIE 2018)

Anais dos Workshops do VII Congresso Brasileiro de Informática na Educação (WCBIE 2018)

\section{Conclusão}

Uma vez imerso nos princípios da Pedagogia por Projetos, o aprendizado significativo percorre um movimento cíclico, pautado pela concepção construtivista, em que o aluno constrói, altera, enriquece e diversifica os esquemas cognitivos. Deve-se os educadores atentar-se para planejamentos didáticos em que esse processo não seja ignorado e sobreposto por sistemas mecânicos, da prática pela prática, da repetição de soluções prontas, sem reflexões, formulações e interações que levam aos desequilíbrios.

Este trabalho teve como mérito principal apresentar um modelo de Projeto como arcabouço didático para mediação da aprendizagem, segundo perspectiva construtivista. Não obstante ao estudo inicial de Programação Orientada a Objetos, a generalização do método para outros contextos educacionais é um campo para novas pesquisas, a fim de corroborar com práticas pedagógicas colaborativas, criativas e ativas.

\section{Referências}

Bittencourt, R.A.; Rodrigues, C.A., Cruz, D.S.S. (2013). Uma Experiência Integrada de Programaçao Orientada a Objetos, Estruturas de Dados e Projeto de Sistemas com PBL. In XXI Workshop sobre Educação em Computação - XXXIII Congresso da Sociedade Brasileira de Computação. SBC.

Borges, K.S.; Menezes, C.S.; Fagundes, L.D.C. (2017). The use of computational thinking in digital fabrication projects a case study from the cognitive perspective. In 2017 IEEE Frontiers in Education Conference (FIE) (pp. 1-6). IEEE.

Carvalho, M.J.S.; Nevado, R.A.; Menezes, C.S. (2005). Arquiteturas pedagógicas para educação à distância: concepções e suporte telemático. In Simpósio Brasileiro de Informática na Educação (Vol. 1, No. 1, pp. 351-360).

Fagundes, L.D.C.; Nevado, R.A.; Basso, M.V.; Bitencourt, J.; Menezes, C.S.; Monteiro, V.C.P. (2006). Projetos de Aprendizagem - uma experiência mediada por ambientes telemáticos. Brazilian Journal of Computers in Education.

Freire, P. (1998) Pedagogia da Autonomia: saberes necessários à prática educativa. Rio de Janeiro: Paz e Terra.

Human-Vogel, S.; Bouwer, C. (2005). Creating a complex learning environment for the mediation of knowledge construction in diverse educational settings. South African Journal of Education (pp. 229-238).

Medeiros, F.P.A.; Santos Jr, P.S.; Bender, M.; Menegussi, L.; Curcher, M. (2017). A Blended Learning Experience Applying Project-Based Learning in an Interdisciplinary Classroom. In: 10th annual International Conference of Education, Research and Innovation - ICERI.

Nunes, R.R.; Pedrosa, D.; Morgado, L.; Martins, P.; Paredes, H.; Cravino, J.; Barreira, C. (2017). SimProgramming: uma abordagem motivacional para a aprendizagem de alunos intermediários de programação. In Anais dos Workshops do Congresso Brasileiro de Informática na Educação (Vol. 6, No. 1, p. 1099).

Piaget, J. (1929-1979). Conjunto das obras publicadas. Université de Génève. Presses Universitaires de France.

Vygotsky, L.S. (1978). Mind in society: The development of higher psychological processes. Harvard university press. 